\title{
A CONCAVE MAXIMIZATION PROBLEM WITH DOUBLE LAYERS OF CONSTRAINTS ON THE TOTAL AMOUNT OF RESOURCES
}

\author{
Ryusuke Hohzaki Koji lida \\ National Defense Academy
}

(Received November 25, 1998; Final July 2, 1999)

\begin{abstract}
This paper investigates a kind of resource allocation problem which maximizes a strictly concave objective function with double layers of constraints on the total amount of resources. Resources are distributed on a two-dimensional space, say, a geographical space with time flow, and are doubly constrained in the sense that the total amount is limited on the whole space and the subtotal amount is constrained at each time too. We derive necessary and sufficient conditions for an optimal solution and propose two methods of solving it. Both methods manipulate Lagrange multipliers and make a sequence of feasible solutions that ultimately satisfy necessary and sufficient conditions for optimality. It is shown by numerical computation that the proposed methods are faster than other well-known methods.
\end{abstract}

\section{Introduction}

This paper investigates a kind of two-dimensional resource allocation problem with constraints doubly layered on the total amount of resources and proposes new methods to solve the problem. The resource allocation problem originates from the search problem studied by Koopman[7] first. He considers the problem of distributing search effort on a continuous space so as to maximize the detection probability of a stationary target and formulates it as a problem of analytically finding an optimal density function of search effort. His study was followed by J. de Guenin[2] who generalized Koopman's work and derived some optimality conditions by a variational method. Generally speaking, it is difficult to analytically obtain an optimal function. When we deal with complicated problems, we often divide a search space into many small regions and numerically obtain solutions with the help of computers. The problem of distributing search effort on the continuous space is converted to a discrete-search-space version. The search problem for a stationary target on the discrete search space is well-formulated as a problem of optimizing a separable function and is easily solved by the Lagrangean multiplier method.

The resource allocation problem comes from the search problem as stated above and hence its main purpose is to optimize the separable function with a constraint on the total amount of resources as Ibaraki and Katoh[5] show. On the other hand, in fields of non-linear programming and global optimization which Konno[6], Mangasarian[8], Martos[9], Horst et al.[3] and Horst and Tuy[4] have contributed to, more generalized problems and methods are mainly discussed. In this paper, we investigate a problem that is more generalized than most resource allocation problems in the sense that the objective function has a more general concave form and the total amount of resources are doubly constrained on a two-dimensional space, but which is also more specialized than some general convex programming problems. The problem is to maximize a concave objective function under constraints doubly layered 
on the weighted total amount of resources. This type of problem has abundant practical applications.

We formulate the problem in the next section. In Section 3, necessary and sufficient conditions for an optimal solution are derived by introducing Lagrange multipliers and the relation between constraints of the problem and the multipliers is elucidated. In Section 4 , two algorithms are proposed for an optimal solution and their validity is proved. In Section 5 , by some examples, we investigate some properties of the optimal solution and examine the computational efficiency of the proposed methods.

\section{Description and Formulation of the Problem}

(AS1) We distribute resources on a two-dimensional discrete space. As a representative case, we take the space consisting of a geographical space of $K$ cells and a time space of $T$ time points which are denoted by $\boldsymbol{K}=\{1, \cdots, K\}$ and $\boldsymbol{T}=$ $\{1, \cdots, T\}$, respectively.

(AS2) Let $\varphi(i, t)$ be the amount of resources distributed on $(i, t) \in \boldsymbol{K} \times \boldsymbol{T}$. It is assumed that $\varphi(i, t)$ is non-negative and limited by an upper bound $m_{i t}>0$ which could be $+\infty$. The distribution of $\varphi(i, t)$ brings cost $c_{i t} \varphi(i, t)$ where $c_{i t}>0$. At each time point $t \in \boldsymbol{T}$, the upper bound of the cost-expenditure is given by $\Phi(t)>0$ and on the whole space, a non-negative real number $M$ is given as the limit of the total amount of cost. We call the constraints imposed at time points and on the whole space the doubly layered constraints.

(AS3) For a distribution plan $\varphi=\{\varphi(i, t), i \in \boldsymbol{K} \quad t \in \boldsymbol{T}\}$, we gain reward $f(\varphi)$. The function $f(\varphi)$ is assumed to be two times continuously differentiable and strictly concave and furthermore bounded on the feasible region determined by (AS2), which is mathematically defined later by inequations (2)-(4).

The purpose of the problem is to maximize the reward function $f(\varphi)$ under the above assumptions. We can flexibly transform the basic model to other ones by relaxing constraints, e.g. $m_{i t}=\infty$ or $\Phi(t)=\infty$. The problem is formulated as a concave maximization problem, which is equivalent to a convex minimization problem.

$$
P_{M}: \max _{\varphi} f(\varphi)
$$

s.t.

$$
\begin{aligned}
& 0 \leq \varphi(i, t) \leq m_{i t}, \quad i \in \boldsymbol{K}, \quad t \in \boldsymbol{T} \\
& \sum_{i=1}^{K} c_{i t} \varphi(i, t) \leq \Phi(t), t \in \boldsymbol{T} \\
& \sum_{t=1}^{T} \sum_{i=1}^{K} c_{i t} \varphi(i, t) \leq M .
\end{aligned}
$$

In the case that $\sum_{i=1}^{K} c_{i t} m_{i t} \leq \Phi(t)$, the limit $\Phi(t)$ is not useful and in the case of $\sum_{t=1}^{T} \Phi(t) \leq$ $M, M$ is not necessary to the problem. Therefore, we assume the following inequalities without loss of generality.

$$
\begin{aligned}
& \sum_{i=1}^{K} c_{i t} m_{i t}>\Phi(t), t \in \boldsymbol{T} \\
& \sum_{t=1}^{T} \Phi(t)>M .
\end{aligned}
$$

If $f(0) \neq 0$, we can regard the problem as the maximization of a new objective function $g(\varphi) \equiv f(\varphi)-f(0)$. For this reason, we assume $f(0)=0$. 
Many researchers have studied special cases of this problem. In the typical resource allocation problem, the maximization of the concave and separable function is studied under constraints (2) and (4), which can be redefined on the one-dimensional space. The separability of the objective function brings many fruitful results: the polynomial-time algorithm for the optimal solution and so on[5]. In search theory, there have been some studies[1, 11] of the concave maximization problem with constraints (2) and (3). In these studies, separability in time permits use of a solution method that repeats the polynomial-time algorithm proposed for the typical resource allocation problem at each time $t$. The more general form of the objective function and the additional constraint (4) on the total amount of resources make $P_{M}$ harder.

\section{Necessary and Sufficient Conditions for Optimality}

Since problem $P_{M}$ is to maximize a strictly concave objective function on a closed convex feasible region, it has a unique optimal solution. Here we derive necessary and sufficient conditions for optimality by introducing some Lagrange multipliers and elucidate the relation between the constraints of the problem and the multipliers.

\subsection{Necessary and sufficient conditions}

Let $\Psi$ be a feasible region fulfilling constraints (2) (4).

Theorem 1 (Necessary and Sufficient Conditions) The feasible solution $\varphi \in \Psi$ which satisfies the following conditions is optimal.

There exist non-negative multipliers $\lambda, \nu_{t}, t=1, \cdots, T$ which guarantee for every $(i, t) \in$ $\boldsymbol{K} \times \boldsymbol{T}$,

$$
\begin{aligned}
& \text { if } \varphi(i, t)=0, \frac{1}{c_{i t}} \frac{\partial f(\varphi)}{\partial \varphi(i, t)} \leq \lambda+\nu_{t} \\
& \text { if } 0<\varphi(i, t)<m_{i t}, \frac{1}{c_{i t}} \frac{\partial f(\varphi)}{\partial \varphi(i, t)}=\lambda+\nu_{t} \\
& \text { if } \varphi(i, t)=m_{i t}, \quad \frac{1}{c_{i t}} \frac{\partial f(\varphi)}{\partial \varphi(i, t)} \geq \lambda+\nu_{t}
\end{aligned}
$$

and for every $t \in \boldsymbol{T}$,

$$
\text { if } \nu_{t}>0, \quad \sum_{i=1}^{K} c_{i t} \varphi(i, t)=\Phi(t)
$$

furthermore,

$$
\text { if } \quad \lambda>0, \quad \sum_{t=1}^{T} \sum_{i=1}^{K} c_{i t} \varphi(i, t)=M .
$$

Proof: Let consider a Lagrangean function with multipliers $\lambda, \nu_{t}, \eta_{i t}^{1}$ and $\eta_{i t}^{2}, i=$ $1, \cdots, K, t=1, \cdots, T$ :

$$
\begin{aligned}
L\left(\varphi ; \lambda, \nu_{t}, \eta_{i t}^{1}, \eta_{i t}^{2}\right)= & f(\varphi)+\lambda\left(M-\sum_{t=1}^{T} \sum_{i=1}^{K} c_{i t} \varphi(i, t)\right)+\sum_{t=1}^{T} \nu_{t}\left(\Phi(t)-\sum_{i=1}^{K} c_{i t} \varphi(i, t)\right) \\
& +\sum_{t=1}^{T} \sum_{i=1}^{K} \eta_{i t}^{1} \varphi(i, t)+\sum_{t=1}^{T} \sum_{i=1}^{K} \eta_{i t}^{2}\left(m_{i t}-\varphi(i, t)\right) .
\end{aligned}
$$

Then we have conditions (7)-(11) from the Kuhn-Tucker conditions[10]. Q.E.D.

Now let us define function $\partial f / \partial \varphi(i, t) / c_{i t}$ by $\rho_{i t}\left(\varphi(i, t) ; \varphi_{-i t}\right)$ where $\varphi_{-i t}$ denotes a vector consisting of all other variables except $\varphi(i, t)$. From the strict concavity of $f(\cdot)$, the function $\rho_{i t}\left(x ; \varphi_{-i t}\right)$ is monotone decreasing for $x$, equation $\rho_{i t}\left(x ; \varphi_{-i t}\right)=y$ has a unique root $x$ that is the inverse function $\rho_{i t}^{-1}\left(y ; \varphi_{-i t}\right)$. Considering the boundedness and differentiability of 
$f(\cdot), \rho_{i t}\left(x ; \varphi_{-i t}\right)$ is well-defined on the domain of $0 \leq x<\infty$. Now we extend the domain of $\rho_{i t}^{-1}(\cdot)$ for computational convenience. Let $a_{i t}(\leq 0)$ be the lower limit of the domain within which $\rho_{i t}\left(x ; \varphi_{-i t}\right)$ has a finite value. If we redefine $\rho_{i t}^{-1}\left(y ; \varphi_{-i t}\right)=a_{i t}$ in the case of $\lim _{x \rightarrow a_{i t}+0} \rho_{i t}\left(x ; \varphi_{-i t}\right)<y$ and $\rho_{i t}^{-1}\left(y ; \varphi_{-i t}\right)=\infty$ in the case of $\lim _{x \rightarrow \infty} \rho_{i t}\left(x ; \varphi_{-i t}\right)>y$, the analytic extension of the domain to $(-\infty, \infty)$ is possible; that is, we have the function $\rho_{i t}^{-1}\left(y ; \varphi_{-i t}\right)$ well-defined on $y \in(-\infty, \infty)$. For example, if we have a concrete expression of $\rho_{i t}\left(x ; \varphi_{-i t}\right)=\exp \left(h\left(\varphi_{-i t}\right) /(x+\alpha)\right)$ where $h\left(\varphi_{-i t}\right)$ is a finite positive-valued function of vector $\varphi_{-i t}$ and $\alpha$ is positive, the inverse function $\rho_{i t}^{-1}\left(y ; \varphi_{-i t}\right)$ becomes $h\left(\varphi_{-i t}\right) / \log y-\alpha$. Though the inverse function can not be defined for all non-positive $y$, the extended definition gives $\rho_{i t}^{-1}\left(y ; \varphi_{-i t}\right)=\infty$ for any $y \leq 1$ and $a_{i t}=-\alpha$. By this extended function, the solution satisfying conditions (7)-(9) can be expressed in the form

$$
\varphi(i, t)=\left[\rho_{i t}^{-1}\left(\lambda+\nu_{t} ; \varphi_{-i t}\right)\right]_{0}^{m_{i t}}
$$

where symbol $[x]_{a}^{b}$ indicates

$$
[x]_{a}^{b}=\left\{\begin{array}{ll}
b, & \text { if } b \leq x \\
x, & \text { if } a<x<b . \\
a, & \text { if } x \leq a
\end{array} .\right.
$$

Using this notation, we can transform constraints (3) and (4) into (14) and (15):

$$
\begin{aligned}
& \sum_{i=1}^{K} c_{i t}\left[\rho_{i t}^{-1}\left(\lambda+\nu_{t} ; \varphi_{-i t}\right)\right]_{0}^{m_{i t}} \leq \Phi(t) \\
& \sum_{t=1}^{T} \sum_{i=1}^{K} c_{i t}\left[\rho_{i t}^{-1}\left(\lambda+\nu_{t} ; \varphi_{-i t}\right)\right]_{0}^{m_{i t}} \leq M .
\end{aligned}
$$

\subsection{Relation between the upper limits of cost and Lagrangean multipliers}

Here we elucidate some relations between the Lagrange multipliers and the local upper limits $\{\Phi(t), t \in \boldsymbol{T}\}$ and the total upper limit $M$. Consider the problems $P_{M_{1}}$ with limits $\left\{M_{1}, \Phi_{1}(t)\right\}$ and $P_{M_{2}}$ with limits $\left\{M_{2}, \Phi_{2}(t)\right\}$ while upper limits $\left\{m_{i t}\right\}$ are assumed to be the same for both problems. Let $\left\{\lambda_{1}, \nu_{1 t}\right\},\left\{\lambda_{2}, \nu_{2 t}\right\}$ be optimal multipliers and $\varphi_{1}, \varphi_{2}$ be optimal solutions for the problems $P_{M_{1}}$ and $P_{M_{2}}$, respectively. If $\varphi_{1} \neq \varphi_{2}$, we have the following relation from the strict concavity of $f(\cdot)$.

$$
f\left(\varphi_{2}\right)<f\left(\varphi_{1}\right)+\sum_{i, t} \frac{\partial f}{\partial \varphi_{1}(i, t)}\left(\varphi_{2}(i, t)-\varphi_{1}(i, t)\right) .
$$

Let $I_{0} \equiv\left\{(i, t) \mid \varphi_{1}(i, t)=0\right\}, I_{1} \equiv\left\{(i, t) \mid 0<\varphi_{1}(i, t)<m_{i t}\right\}$ and $I_{2} \equiv\left\{(i, t) \mid \varphi_{1}(i, t)=\right.$ $\left.m_{i t}\right\}$. From the optimality of $\varphi_{1}$ satisfying conditions (7)-(9), it follows that $\partial f / \partial \varphi_{1}(i, t) \leq$ $c_{i t}\left(\lambda_{1}+\nu_{1 t}\right)$ for $(i, t) \in I_{0}, \partial f / \partial \varphi_{1}(i, t)=c_{i t}\left(\lambda_{1}+\nu_{1 t}\right)$ for $(i, t) \in I_{1}$ and $\partial f / \partial \varphi_{1}(i, t) \geq$ $c_{i t}\left(\lambda_{1}+\nu_{1 t}\right)$ for $(i, t) \in I_{2}$. By subdividing the whole space into $I_{0}, I_{1}$ and $I_{2},(16)$ can be transformed as follows.

$$
\begin{aligned}
& \begin{array}{l}
f\left(\varphi_{2}\right) \\
<
\end{array} \quad f\left(\varphi_{1}\right)+\sum_{(i, t) \in I_{0}} c_{i t}\left(\lambda_{1}+\nu_{1 t}\right) \varphi_{2}(i, t)+\sum_{(i, t) \in I_{1}} c_{i t}\left(\lambda_{1}+\nu_{1 t}\right)\left(\varphi_{2}(i, t)-\varphi_{1}(i, t)\right) \\
& \quad+\sum_{(i, t) \in I_{2}} c_{i t}\left(\lambda_{1}+\nu_{1 t}\right)\left(\varphi_{2}(i, t)-m_{i t}\right) \\
& =f\left(\varphi_{1}\right)+\sum_{i, t} c_{i t}\left(\lambda_{1}+\nu_{1 t}\right)\left(\varphi_{2}(i, t)-\varphi_{1}(i, t)\right) \\
& =f\left(\varphi_{1}\right)+\lambda_{1}\left(\sum_{i, t} c_{i t} \varphi_{2}(i, t)-\sum_{i, t} c_{i t} \varphi_{1}(i, t)\right)+\sum_{t} \nu_{1 t}\left(\sum_{i} c_{i t} \varphi_{2}(i, t)-\sum_{i} c_{i t} \varphi_{1}(i, t)\right) .
\end{aligned}
$$


Noting that $\sum_{i, t} c_{i t} \varphi_{1}(i, t)=M_{1}$ if $\lambda_{1}>0$ and $\sum_{i} c_{i t} \varphi_{1}(i, t)=\Phi_{1}(t)$ if $\nu_{1 t}>0$ from the complementary slackness, we can transform the above expression to

$$
\begin{aligned}
& =f\left(\varphi_{1}\right)+\lambda_{1}\left(\sum_{i, t} c_{i t} \varphi_{2}(i, t)-M_{1}\right)+\sum_{\left\{t \mid \nu_{1 t}>0\right\}} \nu_{1 t}\left(\sum_{i} c_{i t} \varphi_{2}(i, t)-\Phi_{1}(t)\right) \\
& \leq f\left(\varphi_{1}\right)+\lambda_{1}\left(M_{2}-M_{1}\right)+\sum_{t} \nu_{1 t}\left(\Phi_{2}(t)-\Phi_{1}(t)\right) .
\end{aligned}
$$

We finally obtain an important inequality.

$$
f\left(\varphi_{2}\right)<f\left(\varphi_{1}\right)+\lambda_{1}\left(M_{2}-M_{1}\right)+\sum_{t} \nu_{1 t}\left(\Phi_{2}(t)-\Phi_{1}(t)\right) .
$$

Now we are ready to describe some relations between the Lagrange multipliers and the limits of cost.

Lemma 1 There are the following relations between the total limit of cost $M$ and the Lagrange multiplier $\lambda$.

(i) When $\lambda$ approaches $\infty$, an optimal solution becomes $\varphi=\{0, \cdots, 0\}$. That is, $\lambda \rightarrow \infty$ corresponds to the limit of $M=0$.

(ii) When two problems $P_{M_{1}}, P_{M_{2}}$ where only the total limits $M_{1}$ and $M_{2}$ are different have optimal multipliers $\lambda_{1}>0$ and $\lambda_{2}>0$, respectively, $\lambda_{1}<\lambda_{2}$ if $M_{1}>M_{2}$ and vice versa.

(iii) Assume that problem $P_{M}$ has optimal multiplier $\lambda^{*}=0$ and the weighted total amount of optimal solution $\varphi^{*}$ is $C=\sum_{i, t} c_{i t} \varphi^{*}(i, t)$. For arbitrary limit $M^{\prime} \geq C, \varphi^{*}$ and $\lambda^{*}=0$ remain optimal for problem $P_{M^{\prime}}$. The number $C$ gives the minimum of the weighted total amount of resources corresponding to the multiplier $\lambda^{*}=0$. For this reason, we call $C$ the marginal limit of resources.

Proof: (i) When $\lambda$ approaches $\infty$ and $\nu_{t}$ is finite, $\rho_{i t}^{-1}\left(\lambda+\nu_{t} ; \varphi_{-i t}\right)=a_{i t} \leq 0$ holds for all $(i, t)$ and the assertion follows from (13).

(ii) Suppose that problems $P_{M_{1}}, P_{M_{2}}$ have optimal solutions $\varphi_{1}, \varphi_{2}$ and optimal multipliers $\lambda_{1}, \lambda_{2}$, respectively. Since $\lambda_{1}, \lambda_{2}>0, M_{1}=\sum_{i, t} c_{i t} \varphi_{1}(i, t) \neq M_{2}=\sum_{i, t} c_{i t} \varphi_{2}(i, t)$ and therefore $\varphi_{1} \neq \varphi_{2}$. By applying $\Phi_{1}(t)=\Phi_{2}(t)$ to inequality $(17)$, we obtain $f\left(\varphi_{2}\right)<$ $f\left(\varphi_{1}\right)+\lambda_{1}\left(M_{2}-M_{1}\right)$. Similarly we have $f\left(\varphi_{1}\right)<f\left(\varphi_{2}\right)+\lambda_{2}\left(M_{1}-M_{2}\right)$ and consequently the following relation holds.

$$
\lambda_{1}\left(M_{1}-M_{2}\right)<f\left(\varphi_{1}\right)-f\left(\varphi_{2}\right)<\lambda_{2}\left(M_{1}-M_{2}\right)
$$

It tells us that $\lambda_{1}<\lambda_{2}$ if $M_{1}>M_{2}$ and $\lambda_{1}>\lambda_{2}$ if $M_{1}<M_{2}$.

(iii) Let $\varphi^{\prime *}$ and $\lambda^{\prime *}$ be optimal solution and optimal multiplier for the problem $P_{M^{\prime}}$. Since $C \leq M^{\prime}, f\left(\varphi^{*}\right) \leq f\left(\varphi^{*}\right)$. Assuming $\varphi^{*} \neq \varphi^{*}$, we have $f\left(\varphi^{*}\right)<f\left(\varphi^{*}\right)$ by substituting $\varphi_{1}=$ $\varphi^{*}, \varphi_{2}=\varphi^{\prime *}, \Phi_{1}(t)=\Phi_{2}(t)$ and $\lambda_{1}=0$ into inequality $(17)$. It contradicts $f\left(\varphi^{*}\right) \leq f\left(\varphi^{*}\right)$. Therefore $\varphi^{*}=\varphi^{\prime *}$. Q.E.D.

Similarly we can obtain the relation between the local limit $\Phi(t)$ and the multiplier $\nu_{t}$ at time point $t \in \boldsymbol{T}$.

Lemma 2 There are the following relations between the local limit of resources $\Phi(t)$ and Lagarange multiplier $\nu_{t}$ at time point $t \in \boldsymbol{T}$.

(i) When $\nu_{t}$ approaches $\infty$, an optimal solution at time $t$ becomes $\{\varphi(i, t)=0, i \in \boldsymbol{K}\}$. That is, $\nu_{t} \rightarrow \infty$ corresponds to the local limit of $\Phi(t)=0$.

(ii) When two problems where the local limits $\Phi_{1}(t)$ and $\Phi_{2}(t)$ are different only at time $t$ have optimal multipliers $\nu_{1 t}>0$ and $\nu_{2 t}>0$, respectively, $\nu_{1 t}<\nu_{2 t}$ if $\Phi_{1}(t)>\Phi_{2}(t)$ and vice versa.

(iii) Assume that problem $P_{M}$ with the local limit of resources $\Phi(t)$ has optimal multiplier $\nu_{t}^{*}=0$ and the local weighted amount of optimal solution $\varphi^{*}$ at time $t$ is 
$D=\sum_{i} c_{i t} \varphi^{*}(i, t)$. For the problem with arbitrary local limit $\Phi^{\prime}(t) \geq D, \varphi^{*}$ and $\nu_{t}^{*}=0$ remain optimal. The real number $D$ gives the minimum of the local weighted amount of resources at time $t$ corresponding to the multiplier $\nu_{t}^{*}=0$. For this reason, we call $D$ the marginal local limit of resources at time $t$.

As seen from Lemma 1 (ii), the correspondence between $\lambda$ and $M$ varying on $[0, \infty)$ is one-to-one in a part of the half line $[0, \infty)$. The following theorem describes a similar correspondence between $\Phi(t)$ and $\nu_{t}$.

Theorem 2 (i) The necessary and sufficient condition of the marginal limit being 0 is that

$$
\left.\frac{\partial f}{\partial \varphi(i, t)}\right|_{\varphi=0} \leq 0
$$

for every $(i, t) \in \boldsymbol{K} \times \boldsymbol{T}$. Otherwise, the marginal limit $M_{\max }$ corresponding to $\lambda=0$ is given by the weighted total distribution in the following problem.

$$
R P T \text { : } \max _{\varphi} f(\varphi)
$$

s.t.

$$
\begin{aligned}
& 0 \leq \varphi(i, t) \leq m_{i t}, \quad i \in \boldsymbol{K}, \quad t \in \boldsymbol{T} \\
& \sum_{i=1}^{K} c_{i t} \varphi(i, t) \leq \Phi(t), \quad t \in \boldsymbol{T} .
\end{aligned}
$$

Specifically, letting $\varphi^{*}$ be the optimal solution of the problem RPT, the marginal limit is $M_{\text {max }}=\sum_{i, t} c_{i t} \varphi^{*}(i, t)$. Using a new definition of $\lambda_{\text {max }} \equiv \sup _{i, t}\left\{\xi \mid \rho_{i t}^{-1}(\xi ; 0)=0\right\}$, there is the following relationship between the total limit $M$ and the optimal multiplier $\lambda$ for problem $P_{M}$.

The multiplier $\lambda=0$ corresponds to arbitrary $M$ of $M_{\max } \leq M$ and the limit $M=0$ corresponds to arbitrary $\lambda$ of $\lambda_{\max } \leq \lambda$. The multipliers $\lambda_{1}$ and $\lambda_{2}$ of $0<\lambda_{1}<\lambda_{2}<$ $\lambda_{\max }$ correspond to $M_{1}$ and $M_{2}$ of $M_{\max }>M_{1}>M_{2}>0$, respectively, which indicates the monotonic one-to-one correspondence on $0<\lambda<\lambda_{\max }$ and $M_{\max }>M>0$.

(ii) The necessary and sufficient condition of the marginal local limit being $\Phi_{\max }(\tau)=0$ at time $\tau$ is that, given optimal solution $\left\{\varphi^{*}(i, t), i \in \boldsymbol{K}, \tau \neq t \in \boldsymbol{T}\right\}$ at all other time points except $\tau$,

$$
\frac{\partial f}{\partial \varphi(i, \tau)} \mid \begin{array}{ll}
\varphi(i, \tau)=0 \\
\varphi(i, t)=\varphi^{*}(i, t)(t \neq \tau)
\end{array} \quad \leq 0
$$

for every $i \in \boldsymbol{K}$. Otherwise, the marginal local limit $\Phi_{\max }(\tau)$ corresponding to $\nu_{\tau}=0$ is given by the local weighted distribution at time $\tau$ for the following problem.

s.t.

$$
R P L_{\tau}: \max _{\varphi} f(\varphi)
$$

$$
\begin{aligned}
& 0 \leq \varphi(i, t) \leq m_{i t}, \quad i \in \boldsymbol{K}, \quad t \in \boldsymbol{T} \\
& \sum_{i=1}^{K} c_{i t} \varphi(i, t) \leq \Phi(t), \quad \tau \neq t \in \boldsymbol{T} \\
& \sum_{t=1}^{T} \sum_{i=1}^{K} c_{i t} \varphi(i, t) \leq M .
\end{aligned}
$$

Specifically, letting $\varphi$ be the optimal solution of the problem $R P L_{\tau}$, the marginal local limit is given by $\Phi_{\max }(\tau)=\sum_{i} c_{i \tau} \varphi(i, \tau)$. We make a new feasible solution $\varphi^{0}$ by adding $\{\varphi(i, \tau)=0, i \in \boldsymbol{K}\}$ at time $\tau$ to optimal solution $\{\varphi(i, t), i \in \boldsymbol{K}, \tau \neq t \in \boldsymbol{T}\}$ at any other time points except $\tau$ and define $\nu_{\max }(\tau) \equiv \sup _{i}\left\{\xi \mid \rho_{i \tau}^{-1}\left(\lambda+\xi ; \varphi_{-i \tau}^{0}\right)=0\right\}$. 
Then there is the following relationship between the local limit $\Phi(\tau)$ and the optimal multiplier $\nu_{\tau}$ for problem $P_{M}$.

The multiplier $\nu_{\tau}=0$ corresponds to arbitrary $\Phi(\tau)$ of $\Phi_{\max }(\tau) \leq \Phi(\tau)$ and the limit $\Phi(\tau)=0$ corresponds to arbitrary $\nu_{\tau}$ of $\nu_{\max }(\tau) \leq \nu_{\tau}$. The multipliers $\nu_{1 \tau}$ and $\nu_{2 \tau}$ of $0<\nu_{1 \tau}<\nu_{2 \tau}<\nu_{\max }(\tau)$ correspond to $\Phi_{1}(\tau)$ and $\Phi_{2}(\tau)$ of $\Phi_{\max }(\tau)>\Phi_{1}(\tau)>$ $\Phi_{2}(\tau)>0$, respectively, which indicates the monotonic one-to-one correspondence on $0<\nu_{\tau}<\nu_{\max }(\tau)$ and $\Phi_{\max }(\tau)>\Phi(\tau)>0$.

Proof: First we prove part (i). The marginal limit $M_{\max }=0$ means that the optimal solution becomes $\varphi=0$ for any $M>0$. If $\partial f / \partial \varphi(i, t) \leq 0, f(\varphi)<f(0)+\nabla f(0) \cdot \varphi \leq 0$ holds for any $0 \neq \varphi \in \Psi$, which indicates that the optimal solution is $\varphi=0$. Conversely, if $\partial f /\left.\partial \varphi(i, t)\right|_{\varphi=0}>0$ is possible for some $(i, t)$, a small increase of $\varphi(i, t)$ makes the objective function value positive. We have verified the necessary and sufficient condition of $M_{\max }=0$. In this case, it is self-evident that $\lambda=0$ and $\nu_{t}=0$ are optimal.

In the case that $\partial f /\left.\partial \varphi(i, t)\right|_{\varphi=0} \leq 0$ does not hold, we prove that the marginal limit is given by the weighted total amount $M_{\max }$ of the optimal solution of problem $R P T$. Let $\varphi_{M}$ and $f_{M}$ be the optimal solution and the optimal value of $P_{M}$, respectively, and let $\varphi^{*}$ and $f^{*}$ be those of $R P T$. First $f^{*}=f_{M_{\max }}$ is clear. For $M_{\max } \leq M, f^{*}=f_{M_{\max }} \leq f_{M}$ is valid. Considering that the problem $R P T$ is made by deleting a constraint on the total amount of cost from $P_{M}, f_{M} \leq f^{*}$ follows. Now we have $f_{M}=f^{*}=f_{M_{m a x}}$. For $M<M_{\max }$, the assumption of $f_{M}=f_{M_{\max }}$ leads us to $\varphi_{M}=\varphi^{*}$ from the uniqueness of the optimal solution, which means that the weighted total amount of $\varphi_{M}$ is $M_{\text {max }}$. This contradicts that $\varphi_{M}$ has the limit $M$ on the total amount. Therefore, we have $f_{M}<f_{M_{\text {max }}}$. Next we prove that the optimal multiplier of $P_{M_{\max }}$ is $\lambda=0$. The necessary and sufficient conditions for the optimal solution of $R P T$ are given by the following conditions in analogy to Theorem 1:

There exist the non-negative multipliers $\left\{\nu_{t}, t \in \boldsymbol{T}\right\}$ satisfying

$$
\begin{aligned}
& \text { if } \varphi(i, t)=0, \frac{1}{c_{i t}} \frac{\partial f(\varphi)}{\partial \varphi(i, t)} \leq \nu_{t} \\
& \text { if } 0<\varphi(i, t)<m_{i t}, \frac{1}{c_{i t}} \frac{\partial f(\varphi)}{\partial \varphi(i, t)}=\nu_{t} \\
& \text { if } \varphi(i, t)=m_{i t}, \frac{1}{c_{i t}} \frac{\partial f(\varphi)}{\partial \varphi(i, t)} \geq \nu_{t}
\end{aligned}
$$

and for $t=1, \cdots, T$,

$$
\text { if } \nu_{t}>0, \sum_{i=1}^{K} c_{i t} \varphi(i, t)=\Phi(t)
$$

Comparing with the above conditions and conditions (7)-(11) for the problem $P_{M_{\max }}$, we easily know $\lambda=0$. From the above results of $f_{M}<f_{M_{\max }}$ for $M<M_{\max }, f_{M}=f_{M_{\max }}$ for $M_{\max } \leq M$ and the optimal multiplier $\lambda=0$, we conclude that $M_{\max }$ gives the marginal limit.

Next we prove that the optimal multiplier $\lambda$ corresponding to $M=0$ must fulfill $\lambda_{\max } \leq \lambda$. Since the optimal solution must be $\varphi^{0}=0$ in the case of $M=0, \sum_{i} c_{i t} \varphi^{0}(i, t)=0<\Phi(t)$ and hence $\nu_{t}=0$ from condition (10). Now we have $\varphi^{0}(i, t)=\left[\rho_{i t}^{-1}(\lambda ; 0)\right]_{0}^{m_{i t}}$ as the representation of the optimal solution. From the monotone non-increasingness of $\rho_{i t}^{-1}(\cdot)$ and the definition of $\lambda_{\max }, \varphi^{0}(i, t)=0$ holds for $\lambda_{\max } \leq \lambda$. For $\lambda<\lambda_{\max }, \varphi^{0}(i, t)=\rho_{i t}^{-1}(\lambda ; 0)>\rho_{i t}^{-1}\left(\lambda_{\max } ; 0\right)=0$ holds on a certain point $(i, t)$, which contradicts $M=0$. Now we conclude that $\lambda_{\max } \leq \lambda$. From Lemma 1 (ii) and the preceding discussion, it has been verified that $0<\lambda_{1}<\lambda_{2}<$ $\lambda_{\max }$ implies $M_{\max }>M_{1}>M_{2}>0$. The proof of the part (ii) can be done similarly by using Lemma 2. Q.E.D. 


\section{Methods for Optimal Solution}

\subsection{Derivation of optimal multipliers}

Assume that we have the optimal solution $\varphi^{*}=\left\{\varphi^{*}(i, t), i \in \boldsymbol{K} t \in \boldsymbol{T}\right\}$ for problem $P_{M}$. Here we consider a procedure to derive the optimal Lagrange multipliers from $\varphi^{*}$. We define a function of $y \geq 0$ and $\varphi \in \Psi$ which indicates the subtotal amount of cost at time $t$.

$$
S_{t}(y, \varphi) \equiv \sum_{i=1}^{K} c_{i t}\left[\rho_{i t}^{-1}\left(y ; \varphi_{-i t}\right)\right]_{0}^{m_{i t}}
$$

This is a monotone non-increasing continuous function of $y$. Since $\rho_{i t}^{-1}\left(y ; \varphi_{-i t}\right)=a_{i t} \leq 0$ in the limit of $y \rightarrow \infty$, we have $\lim _{y \rightarrow \infty} S_{t}(y, \varphi)=0$. In the limit of $y \rightarrow-\infty, \rho_{i t}^{-1}\left(y ; \varphi_{-i t}\right)=\infty$ and then $\lim _{y \rightarrow-\infty} S_{t}(y, \varphi)=\sum_{i} c_{i t} m_{i t}$. Therefore, $S_{t}(y, \varphi)$ is continuous and monotone decreasing on the range of values of $\left(0, \sum_{i} c_{i t} m_{i t}\right)$ and there exists a unique root $\xi(t, \varphi) \in$ $(-\infty, \infty)$ for the equation $S_{t}(\xi(t, \varphi), \varphi)=\Phi(t)$ with $0<\Phi(t)<\sum_{i} c_{i t} m_{i t}$. Now we sort $\left\{\xi\left(t, \varphi^{*}\right), t \in \mathbb{T}\right\}$ in the order of values, such as $\xi\left(t_{1}, \varphi^{*}\right) \leq \xi\left(t_{2}, \varphi^{*}\right) \leq \cdots \leq \xi\left(t_{T}, \varphi^{*}\right)$. Suppose $\lambda^{*}<\xi\left(t, \varphi^{*}\right)$. Then since $S_{t}\left(\lambda^{*}, \varphi^{*}\right)>S_{t}\left(\xi\left(t, \varphi^{*}\right), \varphi^{*}\right)=\Phi(t)$, it must be $\nu_{t}^{*}>0$ in order to satisfy $S_{t}\left(\lambda^{*}+\nu_{t}^{*}, \varphi^{*}\right) \leq \Phi(t)$ corresponding to inequality (14) and we have $\lambda^{*}+\nu_{t}^{*}=\xi\left(t, \varphi^{*}\right)$ from condition (10). Suppose $\lambda^{*} \geq \xi\left(t, \varphi^{*}\right)$. If $\nu_{t}^{*}>0, S_{t}\left(\lambda^{*}+\nu_{t}^{*}, \varphi^{*}\right)<$ $S_{t}\left(\lambda^{*}, \varphi^{*}\right) \leq S_{t}\left(\xi\left(t, \varphi^{*}\right), \varphi^{*}\right)=\Phi(t)$ holds which is inconsistent with (10). Therefore $\nu_{t}^{*}=0$. Summarizing the above discussion, we have the following relation among $\lambda^{*}, \nu_{t}^{*}$ and $\xi\left(t, \varphi^{*}\right)$.

$$
\begin{aligned}
& \text { If } \lambda^{*}<\xi\left(t, \varphi^{*}\right), \lambda^{*}+\nu_{t}^{*}=\xi\left(t, \varphi^{*}\right) \text { and } \sum_{i} c_{i t} \varphi^{*}(i, t)=\Phi(t), \\
& \text { if } \lambda^{*} \geq \xi\left(t, \varphi^{*}\right), \nu_{t}^{*}=0 .
\end{aligned}
$$

The multiplier $\lambda^{*}$ must be equal to or greater than $\xi\left(t_{1}, \varphi^{*}\right)$. Otherwise the supposition of $\lambda^{*}<\xi\left(t_{1}, \varphi^{*}\right)$ implies that equality holds in condition (3) at all $t \in \boldsymbol{T}$ from (27) and contradicts the basic assumption (6). Now there is a certain integer $I$ such that $\xi\left(t_{I}, \varphi^{*}\right) \leq$ $\lambda^{*}<\xi\left(t_{I+1}, \varphi^{*}\right)$. From (27) and (28), we obtain $\nu_{t_{1}}^{*}=\cdots=\nu_{t_{I}}^{*}=0$ and $\lambda^{*}+\nu_{t_{k}}^{*}=$ $\xi\left(t_{k}, \varphi^{*}\right), k=I+1, \cdots, T$ and the optimal solution as follows.

$$
\varphi^{*}\left(i, t_{k}\right)= \begin{cases}{\left[\rho_{i t_{k}}^{-1}\left(\lambda^{*} ; \varphi_{-i t_{k}}^{*}\right)\right]_{0}^{m_{i t_{k}}},} & k=1, \cdots, I \\ {\left[\rho_{i t_{k}}^{-1}\left(\xi\left(t_{k}, \varphi^{*}\right) ; \varphi_{-i t_{k}}^{*}\right)\right]_{0}^{m_{i t_{k}}},} & k=I+1, \cdots, T\end{cases}
$$

Now we can define the weighted total distribution by the above representation.

$$
\begin{aligned}
Q(\lambda, \varphi) & \equiv \sum_{k=1}^{\mu_{\lambda}(\varphi)} \sum_{i=1}^{K} c_{i t_{k}}\left[\rho_{i t_{k}}^{-1}\left(\lambda ; \varphi_{-i t_{k}}\right)\right]_{0}^{m_{i t_{k}}}+\sum_{k=\mu_{\lambda}(\varphi)+1}^{T} \sum_{i=1}^{K} c_{i t_{k}}\left[\rho_{i t_{k}}^{-1}\left(\xi\left(t_{k}, \varphi\right) ; \varphi_{-i t_{k}}\right)\right]_{0}^{m_{i t_{k}}} \\
& =\sum_{k=1}^{\mu_{\lambda}(\varphi)} \sum_{i=1}^{K} c_{i t_{k}}\left[\rho_{i t_{k}}^{-1}\left(\lambda ; \varphi_{-i t_{k}}\right)\right]_{0}^{m_{i t_{k}}}+\sum_{k=\mu_{\lambda}(\varphi)+1}^{T} \Phi\left(t_{k}\right)
\end{aligned}
$$

where

$$
\mu_{\lambda}(\varphi) \equiv \max \left\{k \mid \xi\left(t_{k}, \varphi\right) \leq \lambda\right\}
$$

In the case of $\mu_{\lambda}(\varphi)=T$, the second term of (30) is neglected and $Q(\lambda, \varphi)$ also represents the weighted total distribution in the case of $\xi\left(t_{T}, \varphi^{*}\right) \leq \lambda^{*}$. We can derive the optimal multiplier $\lambda^{*}$ from the function $Q(\cdot)$ as follows.

(i) In the case of $Q\left(0, \varphi^{*}\right) \leq M$; If $\lambda^{*}>0, Q\left(\lambda^{*}, \varphi^{*}\right)<Q\left(0, \varphi^{*}\right) \leq M$ holds which has inconsistency with condition (11) and hence $\lambda^{*}=0$.

(ii) In the case of $Q\left(0, \varphi^{*}\right)>M$; We have $\lambda^{*}>0$ from the constraint $Q\left(\lambda^{*}, \varphi^{*}\right) \leq M$ and moreover $Q\left(\lambda^{*}, \varphi^{*}\right)=M$ from (11). Considering $\lim _{\lambda \rightarrow \infty} Q\left(\lambda, \varphi^{*}\right)=0$, we see that there exists a unique $\lambda^{*}$ satisfying $Q\left(\lambda^{*}, \varphi^{*}\right)=M$ on $0<\lambda^{*}<\infty$.

Now we have obtained the procedure of giving the optimal multipliers $\lambda^{*}, \nu_{t}^{*}$ when the optimal solution $\varphi^{*}$ is provided, as follows. 
If $Q\left(0, \varphi^{*}\right) \leq M$, the optimal multiplier is $\lambda^{*}=0$. Otherwise, it is uniquely determined by $Q\left(\lambda^{*}, \varphi^{*}\right)=M$. The other multipliers $\left\{\nu_{t}^{*}\right\}$ are given by $\nu_{t_{k}}^{*}=0$ for $k=1, \cdots, \mu_{\lambda^{*}}\left(\varphi^{*}\right)$ and $\nu_{t_{k}}^{*}=\xi\left(t_{k}, \varphi^{*}\right)-\lambda^{*}$ for $k=\mu_{\lambda^{*}}\left(\varphi^{*}\right)+1, \cdots, T$. Then the optimal solution $\varphi^{*}$ is represented by

$$
\varphi^{*}\left(i, t_{k}\right)=\left\{\begin{array}{ll}
{\left[\rho_{i t_{k}}^{-1}\left(\lambda^{*} ; \varphi_{-i t_{k}}^{*}\right)\right]_{0}^{m_{i t_{k}}},} & k=1, \cdots, \mu_{\lambda^{*}}\left(\varphi^{*}\right) \\
{\left[\rho_{i t_{k}}^{-1}\left(\xi\left(t_{k}, \varphi^{*}\right) ; \varphi_{-i t_{k}}^{*}\right)\right]_{0}^{m_{i t_{k}}},} & k=\mu_{\lambda^{*}}\left(\varphi^{*}\right)+1, \cdots, T
\end{array},\right.
$$

where $T$ time points are sorted in the order of values of $\left\{\xi\left(t, \varphi^{*}\right), t=1, \cdots, T\right\}$, such that $\xi\left(t_{1}, \varphi^{*}\right) \leq \xi\left(t_{2}, \varphi^{*}\right) \leq \cdots \leq \xi\left(t_{T}, \varphi^{*}\right)$ for $t_{1}, t_{2}, \cdots, t_{T}$.

\subsection{Algorithms for optimal solution}

We propose two algorithms for optimal solution of $P_{M}$.

(1) Gradient-completion method

This algorithm varies the feasible solution so as to satisfy Eqs. (10) and (11) and terminates when Eqs. (7)-(9) are fulfilled. The equations (7)-(9) are the condition for the gradient of the Lagrangean function, which is the reason why we call the algorithm the gradient-completion method. The outline of it is written down as follows.

\section{Algorithm GC}

(GC1) If condition (18) is satisfied, terminate. Zero vector is the optimal solution. Otherwise, set $j=0$ and any initial feasible solution $\varphi^{j}$, for example, $\varphi^{0}=$ $\{0, \cdots, 0\}$.

(GC2) Using $\varphi^{j}$, define $\rho_{i t}^{-1}\left(\cdot ; \varphi_{-i t}^{j}\right)$ and obtain a set $T_{0}=\left\{t \in \boldsymbol{T} \mid S_{t}\left(0, \varphi^{j}\right) \leq \Phi(t)\right\}$ and $T^{c}=\boldsymbol{T}-T_{0}$. Let $L$ be the size $\left|T_{0}\right|$ of $T_{0}$. Number elements of $T_{0}$ such as $t_{1}, t_{2}, \cdots, t_{L}$. For $t \in T^{c}$, calculate $\left\{\xi\left(t, \varphi^{j}\right) \mid t \in T^{c}\right\}$ satisfying $S_{t}\left(\xi\left(t, \varphi^{j}\right), \varphi^{j}\right)=$ $\Phi(t)$ and assign each element of $T^{c}$ numbers $t_{L+1}, \cdots, t_{T}$ in the order of $0<$ $\xi\left(t_{L+1}, \varphi^{j}\right) \leq \cdots \leq \xi\left(t_{T}, \varphi^{j}\right)$. Obtain $\lambda$ by the following procedure.

(i) If $Q\left(0, \varphi^{j}\right) \leq M$, set $\lambda=0$.

(ii) If $Q\left(0, \varphi^{j}\right)>M$, calculate a unique $\lambda>0$ for which $Q\left(\lambda, \varphi^{j}\right)=M$.

Generate a new feasible solution $\hat{\varphi}^{j}$ using $\xi\left(t_{k}, \varphi^{j}\right)$ and $\lambda$ as follows.

$$
\widehat{\varphi}^{j}\left(i, t_{k}\right)=\left\{\begin{array}{ll}
{\left[\rho_{i t_{k}}^{-1}\left(\lambda ; \varphi_{-i t_{k}}^{j}\right)\right]_{0}^{m_{i t_{k}}},} & k=1, \cdots, \mu_{\lambda}\left(\varphi^{j}\right) \\
{\left[\rho_{i t_{k}}^{-1}\left(\xi\left(t_{k}, \varphi^{j}\right) ; \varphi_{-i t_{k}}^{j}\right)\right]_{0}^{m_{i t_{k}}},} & k=\mu_{\lambda}\left(\varphi^{j}\right)+1, \cdots, T .
\end{array} .\right.
$$

(GC3) If $\hat{\varphi}^{j}=\varphi^{j}$, terminate. The current $\varphi^{j}$ is the optimal solution. Otherwise, execute the following line search and generate the next feasible solution $\varphi^{j+1}=$ $\varphi^{j}+\theta^{*}\left(\widehat{\varphi}^{j}-\varphi^{j}\right)$.

$$
f\left(\varphi^{j}+\theta^{*}\left(\widehat{\varphi}^{j}-\varphi^{j}\right)\right)=\max _{0<\theta \leq \bar{\theta}} f\left(\varphi^{j}+\theta\left(\widehat{\varphi}^{j}-\varphi^{j}\right)\right),
$$

where $\bar{\theta}=\min \left\{\theta_{1}, \theta_{2}, \theta_{3}, \theta_{4}\right\}$ and $\theta_{1}, \theta_{2}, \theta_{3}, \theta_{4}$ are given by four estimations.

$$
\begin{aligned}
& \theta_{1} \equiv\left\{\begin{array}{l}
\infty, \text { if there is no }(i, t) \text { for which } \widehat{\varphi}^{j}(i, t)-\varphi^{j}(i, t)<0 \\
\min _{i, t}\left\{-\frac{\varphi^{j}(i, t)}{\hat{\varphi}^{j}(i, t)-\varphi^{j}(i, t)} \mid \hat{\varphi}^{j}(i, t)-\varphi^{j}(i, t)<0\right\}, \text { otherwise }
\end{array}\right. \\
& \theta_{2} \equiv\left\{\begin{array}{l}
\infty, \text { if there is no }(i, t) \text { for which } \hat{\varphi}^{j}(i, t)-\varphi^{j}(i, t)>0 \\
\min _{i, t}\left\{\frac{m_{i t}-\varphi^{j}(i, t)}{\hat{\varphi}^{j}(i, t)-\varphi^{j}(i, t)} \mid \widehat{\varphi}^{j}(i, t)-\varphi^{j}(i, t)>0\right\}, \text { otherwise }
\end{array}\right. \\
& \theta_{3} \equiv\left\{\begin{array}{l}
\infty, \text { if there is no } t \text { for which } \sum_{i} c_{i t}\left(\hat{\varphi}^{j}(i, t)-\varphi^{j}(i, t)\right)>0 \\
\min _{t}\left\{\frac{\Phi(t)-\sum_{i} c_{i t} \varphi^{j}(i, t)}{\sum_{i} c_{i t}\left(\hat{\varphi}^{j}(i, t)-\varphi^{j}(i, t)\right)} \mid \sum_{i} c_{i t}\left(\hat{\varphi}^{j}(i, t)-\varphi^{j}(i, t)\right)>0\right\}, \text { otherwise }
\end{array}\right. \\
& \theta_{4} \equiv\left\{\begin{array}{l}
\infty, \text { if } \sum_{i, t} c_{i t}\left(\hat{\varphi}^{j}(i, t)-\varphi^{j}(i, t)\right)<0 \\
\frac{M-\sum_{i, t} c_{i t} \varphi^{j}(i, t)}{\sum_{i, t} c_{i t}\left(\hat{\varphi}^{j}(i, t)-\varphi^{j}(i, t)\right)}, \text { otherwise }
\end{array}\right.
\end{aligned}
$$


Increase $j$ by one, $j=j+1$, and go to (GC2).

The range of $\theta$ in the line search (34) is estimated by considering the feasibility of $\varphi^{\prime}(i, t)=$ $\varphi^{j}(i, t)+\theta\left(\widehat{\varphi}^{j}(i, t)-\varphi^{j}(i, t)\right): 0 \leq \varphi^{\prime}(i, t) \leq m_{i t}, \sum_{i} \varphi^{\prime}(i, t) \leq \Phi(t)$ and $\sum_{i, t} \varphi^{\prime}(i, t) \leq M$. From definitions (35)-(38), all of $\theta_{1}, \theta_{2}, \theta_{3}, \theta_{4}$ are equal to or more than 1 and at least one of them is finite and hence $1 \leq \bar{\theta}<\infty$.

The procedure of (GC2) and the representation (33) are the same as (29) assuming that $\varphi^{j}$ is optimal. By (33), $\widehat{\varphi}^{j}$ is generated so as to satisfy conditions (10) and (11). If $\widehat{\varphi}^{j}=\varphi^{j}$, the conditions (7)-(9) become valid at the same time, which indicates that current solution is optimal.

In the case of $\widehat{\varphi}^{j} \neq \varphi^{j}$ in (GC2), the next solution $\varphi^{j+1}$ is generated. Then if we can clarify that $f\left(\varphi^{j}\right)<f\left(\varphi^{j+1}\right)$, the proof that $\lim _{j \rightarrow \infty} f\left(\varphi^{j}\right)$ converges to an optimal value will be completed from the boundedness of the feasible region $\Psi$ and the finiteness of $f(\cdot)$. Since $\varphi^{j+1}$ is given by the line search in direction $\hat{\varphi}^{j}-\varphi^{j}$, if the direction always becomes an ascent direction, that is, $\nabla f\left(\varphi^{j}\right)\left(\widehat{\varphi}^{j}-\varphi^{j}\right)>0$, we have $f\left(\varphi^{j}\right)<f\left(\varphi^{j+1}\right)$. The assertion of $\nabla f\left(\varphi^{j}\right)\left(\hat{\varphi}^{j}-\varphi^{j}\right)>0$ is made by the mean value theorem and the strict concavity of $f(\cdot)$ below.

$$
\begin{aligned}
& \nabla f\left(\varphi^{j}\right)\left(\widehat{\varphi}^{j}-\varphi^{j}\right)=\left.\sum_{i, t} \frac{\partial f(\varphi)}{\partial \varphi(i, t)}\right|_{\varphi(i, t)=\varphi^{j}(i, t)}\left(\widehat{\varphi}^{j}(i, t)-\varphi^{j}(i, t)\right) \\
& =\sum_{i, t}\left\{\begin{array}{l}
\frac{\partial f(\varphi)}{\partial \varphi(i, t)} \mid \begin{array}{l}
\varphi(i, t)=\widehat{\varphi}^{j}(i, t) \\
\varphi(k, \tau)=\varphi^{j}(k, \tau)((k, \tau) \neq(i, t))
\end{array}
\end{array}\right. \\
& \left.+\left(\varphi^{j}(i, t)-\widehat{\varphi}^{j}(i, t)\right) \frac{\partial^{2} f(\varphi)}{\partial \varphi(i, t)^{2}} \mid \begin{array}{l}
\varphi(i, t)=\left(1-\theta_{i t}\right) \widehat{\varphi}^{j}(i, t)+\theta_{i t} \varphi^{j}(i, t) \\
\varphi(k, \tau)=\varphi^{j}(k, \tau)((k, \tau) \neq(i, t))
\end{array}\right\}\left(\hat{\varphi}^{j}(i, t)-\varphi^{j}(i, t)\right) \\
& =\sum_{i, t}\left(\widehat{\varphi}^{j}(i, t)-\varphi^{j}(i, t)\right) \frac{\partial f(\varphi)}{\partial \varphi(i, t)} \mid \varphi(i, t)=\widehat{\varphi}^{j}(i, t) \\
& \varphi(k, \tau)=\varphi^{j}(k, \tau)((k, \tau) \neq(i, t)) \\
& -\left(\hat{\varphi}^{j}(i, t)-\varphi^{j}(i, t)\right)^{2} \frac{\partial^{2} f(\varphi)}{\partial \varphi(i, t)^{2}} \mid \varphi(i, t)=\left(1-\theta_{i t}\right) \widehat{\varphi}^{j}(i, t)+\theta_{i t} \varphi^{j}(i, t) \\
& \varphi(k, \tau)=\varphi^{j}(k, \tau)((k, \tau) \neq(i, t)) \\
& >\sum_{i, t}\left(\widehat{\varphi}^{j}(i, t)-\varphi^{j}(i, t)\right) \frac{\partial f(\varphi)}{\partial \varphi(i, t)} \mid \varphi(i, t)=\widehat{\varphi}^{j}(i, t) \\
& \varphi(k, \tau)=\varphi^{j}(k, \tau)((k, \tau) \neq(i, t))
\end{aligned}
$$

We subdivide $\boldsymbol{K} \times \boldsymbol{T}$ into $I_{0} \equiv\left\{(i, t) \mid \widehat{\varphi}^{j}(i, t)=0\right\}, I_{1} \equiv\left\{(i, t) \mid 0<\widehat{\varphi}^{j}(i, t)<m_{i t}\right\}$ and $I_{2} \equiv\left\{(i, t) \mid \widehat{\varphi}^{j}(i, t)=m_{i t}\right\}$, and then we can transform the above expression as follows.

$$
\begin{aligned}
& \geq \sum_{(i, t) \in I_{0}} c_{i t}\left(\lambda+\nu_{t}\right)\left(-\varphi^{j}(i, t)\right)+\sum_{(i, t) \in I_{1}} c_{i t}\left(\lambda+\nu_{t}\right)\left(\widehat{\varphi}^{j}(i, t)-\varphi^{j}(i, t)\right) \\
& \quad+\sum_{(i, t) \in I_{2}} c_{i t}\left(\lambda+\nu_{t}\right)\left(m_{i t}-\varphi^{j}(i, t)\right) \\
& =\sum_{i, t} c_{i t}\left(\lambda+\nu_{t}\right)\left(\widehat{\varphi}^{j}(i, t)-\varphi^{j}(i, t)\right) \\
& =\lambda \sum_{i, t}\left(c_{i t} \widehat{\varphi}^{j}(i, t)-c_{i t} \varphi^{j}(i, t)\right)+\sum_{t} \nu_{t} \sum_{i}\left(c_{i t} \widehat{\varphi}^{j}(i, t)-c_{i t} \varphi^{j}(i, t)\right) \\
& \geq 0
\end{aligned}
$$


The last inequality comes from the fact that $\sum_{i, t} c_{i t} \widehat{\varphi}^{j}(i, t)=M$ for $\lambda>0$ and $\sum_{i} c_{i t} \widehat{\varphi}^{j}(i, t)=$ $\Phi(t)$ for $\nu_{t}>0$. Now the proof is completed. The gradient-completion method generates a sequence of feasible solutions $\varphi^{0}, \varphi^{1}, \varphi^{2}, \cdots$ with the increasing values of $f\left(\varphi^{0}\right)<f\left(\varphi^{1}\right)<$ $f\left(\varphi^{2}\right)<\cdots$ and terminates to give an optimal solution.

(2) Total amount-completion method

We propose another algorithm for optimal solution. We know that there is a simple relation between the optimal multiplier $\lambda$ and the weighted total amount of resources as stated in Theorem 2. First we calculate an optimal solution $\varphi^{\lambda *}$ and the marginal limit $M_{\max }$ corresponding to $\lambda=0$. If $M_{\max } \leq M$, the optimal solution of the problem is nothing but $\varphi^{\lambda *}$. If $M_{\max }>M$, the algorithm adjusts $\lambda$ and finds a solution satisfying $\sum_{i, t} c_{i t} \varphi^{\lambda *}(i, t)=M$ at last. The varying of $\lambda$ means the indirect adjustment of the weighted total amount of resources. That is why we call the algorithm the total amount-completion method. Let $\varphi^{*}$ be the optimal solution of the problem. The outline of the algorithm is as follows.

\section{Algorithm TAC}

(TAC1) If condition (18) is satisfied, terminate. Zero vector is the optimal solution. Otherwise, calculate $\lambda_{\text {max }}=\sup _{i, t}\left\{\xi \mid \rho_{i t}^{-1}(\xi ; 0)=0\right\}$.

(TAC2) By a subprocedure $A L_{\lambda}$ which is described later, for $\lambda=0$, calculate an optimal solution $\varphi^{\lambda *}$ and the marginal limit $M_{\max }=\sum_{i, t} c_{i t} \varphi^{\lambda *}(i, t)$.

(i) If $M_{\max } \leq M$, terminate. The current $\varphi^{\lambda *}$ is optimal.

(ii) If $M_{\max }>M$, set $\underline{\lambda}=0$ and $\bar{\lambda}=\lambda_{\max }$.

(TAC3) Update $\lambda$ by $\lambda=(\underline{\lambda}+\bar{\lambda}) / 2$ and calculate $\varphi^{\lambda *}$ by the subprocedure $A L_{\lambda}$.

(i) If $\sum_{i, t} c_{i t} \varphi^{\lambda *}(i, t)=M$, terminate. The current solution $\varphi^{\lambda *}$ is optimal.

(ii) If $\sum_{i, t} c_{i t} \varphi^{\lambda *}(i, t)>M$, set $\underline{\lambda}=\lambda$ and repeat (TAC3).

(iii) If $\sum_{i, t} c_{i t} \varphi^{\lambda *}(i, t)<M$, set $\bar{\lambda}=\lambda$ and repeat (TAC3).

$A L_{\lambda}$ is the subprocedure to give the optimal solution $\varphi^{\lambda *}$ corresponding to the given multiplier $\lambda$.

\section{Algorithm $A L_{\lambda}$}

(AL1) As a tentative solution, take $\varphi$ delivered from the procedure TAC. Substitute $\{\tilde{\varphi}(i, t), i \in \boldsymbol{K}\}$ which is derived after the execution of steps (AL2)-(AL4) at time $t \in \boldsymbol{T}$ for a part $\{\varphi(i, t), i \in \boldsymbol{K}\}$ of $\varphi$. Denote this operation by operator $\Lambda_{t}$. Repeat the operation of $\varphi=\Lambda_{t} \varphi$ for all $t \in \boldsymbol{T}$ and make $\varphi$ converge to a vector which is $\varphi^{\lambda *}$.

(AL2) At a fixed time point $t$, initialize $\nu_{t}=0$ and obtain $\{\varphi(i, t), i \in \boldsymbol{K}\}$ satisfying $\varphi(i, t)=\left[\rho_{i t}^{-1}\left(\lambda+\nu_{t} ; \varphi_{-i t}\right)\right]_{0}^{m_{i t}}$ by a subprocedure $A L_{\lambda}\left(\nu_{t}\right)$ which is described later. If $\sum_{i} c_{i t} \varphi(i, t) \leq \Phi(t)$, terminate. Otherwise estimate $\nu_{t}$ and $\varphi$ satisfying $\sum_{i} c_{i t}\left[\rho_{i t}^{-1}\left(\lambda+\nu_{t} ; \varphi_{-i t}\right)\right]_{0}^{m_{i t}}=\Phi(t)$ by the following steps (AL3) and (AL4).

(AL3) Set $\underline{\nu}=0$ and $\bar{\nu}$ being large enough.

(AL4) Update $\nu_{t}$ by $\nu_{t}=(\underline{\nu}+\bar{\nu}) / 2$ and obtain $\{\varphi(i, t), i \in \boldsymbol{K}\}$ satisfying $\varphi(i, t)=$ $\left[\rho_{i t}^{-1}\left(\lambda+\nu_{t} ; \varphi_{-i t}\right)\right]_{0}^{m_{i t}}$ by a subprocedure $A L_{\lambda}\left(\nu_{t}\right)$.

(i) If $\sum_{i} c_{i t} \varphi(i, t)=\Phi(t)$, terminate.

(ii) If $\sum_{i} c_{i t} \varphi(i, t)>\Phi(t)$, set $\underline{\nu}=\nu_{t}$ and repeat (AL4).

(iii) If $\sum_{i} c_{i t} \varphi(i, t)<\Phi(t)$, set $\bar{\nu}=\nu_{t}$ and repeat (AL4). 
The subprocedure $A L_{\lambda}\left(\nu_{t}\right)$ is a repeating procedure as follows.

Algorithm $A L_{\lambda}\left(\nu_{t}\right)$

Start from current solution $\varphi$ and repeat the substitution of $\left[\rho_{i t}^{-1}\left(\lambda+\nu_{t} ; \varphi_{-i t}\right)\right]_{0}^{m_{i t}}$ for $\varphi(i, t)$ at every $i=1,2, \cdots, K$ until $\varphi$ converges.

The total amount-completion method terminates and then gives an optimal solution $\varphi^{*}$ as the gradient-completion method does. First let us make sure that Algorithm $A L_{\lambda}\left(\nu_{t}\right)$ produces a solution satisfying $\varphi(i, t)=\left[\rho_{i t}^{-1}\left(\lambda+\nu_{t} ; \varphi_{-i t}\right)\right]_{0}^{m_{i t}}$ at time point $t$. The calculation of $\left[\rho_{i t}^{-1}\left(\lambda+\nu_{t} ; \varphi_{-i t}\right)\right]_{0}^{m_{i t}}$ is equivalent to finding a $\varphi(i, t)$ satisfying the following conditions.

$$
\begin{aligned}
& \text { If } \varphi(i, t)=0, \frac{1}{c_{i t}} \frac{\partial f(\varphi)}{\partial \varphi(i, t)} \leq \lambda+\nu_{t}, \\
& \text { if } 0<\varphi(i, t)<m_{i t}, \frac{1}{c_{i t}} \frac{\partial f(\varphi)}{\partial \varphi(i, t)}=\lambda+\nu_{t}, \\
& \text { if } \varphi(i, t)=m_{i t}, \quad \frac{1}{c_{i t}} \frac{\partial f(\varphi)}{\partial \varphi(i, t)} \geq \lambda+\nu_{t} .
\end{aligned}
$$

The conditions are the necessary and sufficient conditions for the optimal solution of the following univariate convex programming problem.

s.t.

$$
L P_{i t}\left(\lambda, \nu_{t}\right): \max _{\varphi(i, t)}\left\{f(\varphi)-\lambda \sum_{j, \tau} c_{j \tau} \varphi(j, \tau)-\sum_{\tau} \nu_{\tau} \sum_{j} c_{j \tau} \varphi(j, \tau)\right\}
$$

$0 \leq \varphi(i, t) \leq m_{i t}$

$\{\varphi(j, t), i \neq j \in \boldsymbol{K}\}$ are given.

$\{\varphi(j, \tau), j \in \boldsymbol{K}, t \neq \tau \in \boldsymbol{T}\}$ are given.

The above objective function which we denote by $\tilde{f}(\varphi)$ is strictly concave and hence the problem has a unique optimal solution. By substituting an optimal solution for only an element $\varphi(i, t)$ of the current tentative solution $\{\varphi(i, t), i \in \boldsymbol{K}\}$ at time $t$, which we denote by operator $\Gamma_{i t}$, it follows that $\tilde{f}(\varphi) \leq \tilde{f}\left(\Gamma_{i t} \varphi\right)$ while equality holds only if $\varphi=\Gamma_{i t} \varphi$. Therefore, the repetition of $\Gamma_{i t}$ for $i \in \boldsymbol{K}$ brings the convergence to a solution $\varphi$ which fulfills $\varphi(i, t)=\left[\rho_{i t}^{-1}\left(\lambda+\nu_{t} ; \varphi_{-i t}\right)\right]_{0}^{m_{i t}}$ for every $i \in \boldsymbol{K}$ at $t$.

In steps (AL3) and (AL4) of Algorithm $A L_{\lambda}$, while fixing $\varphi$ at any other time point except $t,\{\varphi(i, t), i \in \boldsymbol{K}\}$ satisfying the following conditions at $t$ is obtained.

$$
\begin{aligned}
& \varphi(i, t)=\left[\rho_{i t}^{-1}\left(\lambda+\nu_{t} ; \varphi_{-i t}\right)\right]_{0}^{m_{i t}}, i \in \boldsymbol{K} \\
& \text { if } \nu_{t}>0, \quad \sum_{i} c_{i t} \varphi(i, t)=\Phi(t) .
\end{aligned}
$$

These conditions are equivalent to the following conditions as seen by analogy to Theorem 1.

$$
\begin{aligned}
& \text { If } \varphi(i, t)=0, \frac{1}{c_{i t}} \frac{\partial f}{\partial \varphi(i, t)} \leq \lambda+\nu_{t} \\
& \text { if } 0<\varphi(i, t)<m_{i t}, \quad \frac{1}{c_{i t}} \frac{\partial f}{\partial \varphi(i, t)}=\lambda+\nu_{t} \\
& \text { if } \varphi(i, t)=m_{i t}, \quad \frac{1}{c_{i t}} \frac{\partial f}{\partial \varphi(i, t)} \geq \lambda+\nu_{t}
\end{aligned}
$$

for $i \in \boldsymbol{K}$ and

$$
\text { if } \nu_{t}>0, \quad \sum_{i} c_{i t} \varphi(i, t)=\Phi(t) \text {. }
$$

These conditions are necessary and sufficient for an optimal solution of the following convex programming problem $L P_{t}(\lambda)$ with a fixed $t$. 


$$
\begin{aligned}
& L P_{t}(\lambda): \max _{\varphi}\left\{f(\varphi)-\lambda \sum_{i, \tau} c_{i \tau} \varphi(i, \tau)\right\} \\
& \text { s.t. } \\
& \quad 0 \leq \varphi(i, t) \leq m_{i t}, \quad i \in \boldsymbol{K} \\
& \sum_{i} c_{i t} \varphi(i, t) \leq \Phi(t) \\
& \{\varphi(i, \tau), i \in \boldsymbol{K}, t \neq \tau \in \boldsymbol{T}\} \text { are given. }
\end{aligned}
$$

Since the objective function $\widehat{f}(\varphi)=f(\varphi)-\lambda \sum_{i, \tau} c_{i \tau} \varphi(i, \tau)$ is strictly concave and the feasible region is a closed convex set, the problem has a unique optimal solution. By the operation $\Lambda_{t} \varphi,\{\varphi(i, t), i \in \boldsymbol{K}\}$ is changed to $\{\widetilde{\varphi}(i, t), i \in \boldsymbol{K}\}$ which is the optimal solution of problem $L P_{t}(\lambda)$ and hence $\widehat{f}(\varphi) \leq \widehat{f}\left(\Lambda_{t} \varphi\right)$ where equality holds only if $\varphi=\Lambda_{t} \varphi$. By the repetition of $\Lambda_{t}$ for $t=1, \cdots, T$, the solution converges to a solution which is just the optimal solution of the following problem $L P(\lambda)$.

$$
L P(\lambda): \max _{\varphi}\left\{f(\varphi)-\lambda \sum_{i, t} c_{i t} \varphi(i, t)\right\}
$$

s.t.

$$
\begin{aligned}
& 0 \leq \varphi(i, t) \leq m_{i t}, \quad i \in \boldsymbol{K}, t \in \boldsymbol{T} \\
& \sum_{i} c_{i t} \varphi(i, t) \leq \Phi(t), \quad t \in \boldsymbol{T} .
\end{aligned}
$$

In case $\lambda=0$, the problem $L P(\lambda)$ is identical with the problem $R P T$ in Theorem 2 and Algorithm $A L_{\lambda}$ gives the marginal limit $M_{m a x}$ and the corresponding optimal solution $\varphi^{\lambda *}$ in (TAC2). We can say that if $M_{\max } \leq M$ holds in (TAC2), $\varphi^{\lambda *}$ becomes an ultimately optimal solution. In the case of $\lambda>0$, denoting the total cost of the solution of $A L_{\lambda}$ by $M_{\lambda}=$ $\sum_{i, t} c_{i t} \varphi(i, t)$, it follows that $\varphi(i, t)=\left[\rho_{i t}^{-1}\left(\lambda+\nu_{t} ; \varphi_{-i t}\right)\right]_{0}^{m_{i t}}$ for all $(i, t)$ and $\sum_{i} c_{i t} \varphi(i, t)=\Phi(t)$ if $\nu_{t}>0$, for $t \in \boldsymbol{T}$ and moreover $M_{\lambda}=\sum_{i, t} c_{i t} \varphi(i, t)$ if $\lambda>0$. This means that we already have obtained the optimal solution $\varphi^{\lambda *}$ with its total amount $M_{\lambda}$ corresponding to the multiplier $\lambda$. Therefore, just when $\sum_{i, t} c_{i t} \varphi^{\lambda *}(i, t)=M$ occurs by adjusting multiplier $\lambda$, we have obtained an ultimately optimal solution. Now we have proved the validity of the total amount-completion method.

With an additional assumption, only $K$ calculations for $i=1, \cdots, K$ are necessary to Algorithm $A L_{\lambda}\left(\nu_{t}\right)$ instead of repeating the substitution. The assumption is as follows.

(AS4) For all $i \in \boldsymbol{K}, \rho_{i t}^{-1}\left(\xi ; \varphi_{-i t}\right)$ does not contain $\{\varphi(j, t), j \in \boldsymbol{K}\}$ explicitly.

The revising method of $\lambda=(\underline{\lambda}+\bar{\lambda}) / 2$ in (TAC3) or $\nu_{t}=(\underline{\nu}+\bar{\nu}) / 2$ in (AL4) is simply binary search. There could be other ideas for revising the multipliers. We can exploit the monotonicity of the relation between the multipliers and the weighted total distribution explained in Theorem 2. That is, assuming that there is an inversely proportional relation between them, we obtain a new revising method for $\lambda$ in (TAC3) or $\nu_{t}$ in (AL4).

Let $\bar{M}$ and $\underline{M}(\bar{M}>\underline{M})$ be the total cost limits corresponding to $\underline{\lambda}$ and $\bar{\lambda}$, respectively. The new revising of $\lambda$ is as follows.

$$
\lambda=\frac{\bar{M}-M}{\bar{M}-\underline{M}} \bar{\lambda}+\frac{M-\underline{M}}{\bar{M}-\underline{M}} \underline{\lambda} .
$$

Corresponding to $\underline{\lambda}=0$ and $\bar{\lambda}=\lambda_{\max }$, initial setting of $\bar{M}=M_{\max }$ and $\underline{M}=0$ must be added in (TAC2)(ii). Similarly, letting $\overline{\Phi_{t}}$ and $\underline{\Phi_{t}}\left(\overline{\Phi_{t}}>\underline{\Phi_{t}}\right)$ be the local cost limits corresponding to $\underline{\nu}$ and $\bar{\nu}$ at time $t \in \boldsymbol{T}$, respectively, we may revise $\nu_{t}$ in (AL4) by the following estimation.

$$
\nu_{t}=\frac{\bar{\Phi}_{t}-\Phi(t)}{\overline{\Phi_{t}}-\underline{\Phi_{t}}} \bar{\nu}+\frac{\Phi(t)-\underline{\Phi_{t}}}{\overline{\Phi_{t}}-\underline{\Phi_{t}}} \underline{\nu} .
$$




\section{Numerical Examples}

Here we apply the two methods proposed in the previous section to some examples in order to clarify some characteristics of the optimal solution. Furthermore, to elucidate the computational efficiency of the proposed methods, we compare our methods with some well-known methods of non-linear programming with respect to computational time. Let us consider the following search model.

(1) A searcher wants to detect a target by distributing search effort which can be continuously divided on the search space of a discrete cell space and a discrete time space. The cell space and the time space are denoted by $\boldsymbol{K}=\{1, \cdots, K\}$ and $\boldsymbol{T}=\{1, \cdots, T\}$, respectively.

(2) The available search effort has some constraints on the total amount. Let $\varphi(i, t)$ be the effort distributed on a point $(i, t) \in \boldsymbol{K} \times \boldsymbol{T}$. It must be $0 \leq \varphi(i, t) \leq m_{i t}$. The subtotal amounts of effort must not be beyond $\Phi(t)$ and $M$, respectively, that is, $\sum_{i} \varphi(i, t) \leq \Phi(t)$ and $\sum_{i, t} \varphi(i, t) \leq M$.

(3) The target has several possible paths, denoted by $\Omega$. Path $\omega \in \Omega$ is selected with probability $\pi(\omega)$. The path $\omega$ is represented by a sequence of cells according to time flow, $\{\omega(t), t \in \boldsymbol{T}\}$ where $\omega(t)$ is the target's position in the cell space at time $t$. Such path information is given to the searcher in advance. It is assumed that $\sum_{\omega \in \Omega} \pi(\omega)=1$.

(4) By search effort $\varphi(i, t)$ distributed on a point $(i, t) \in \boldsymbol{K} \times \boldsymbol{T}$, the target is detected at time $t$ with probability $1-\exp \left(-\alpha_{i} \varphi(i, t)\right)$ only if $\omega(t)=i$. The positive real number $\alpha_{i}$ indicates the detectability of cell $i$. Events of the detection at each time are assumed to occur independently each other.

In the search model, our purpose is to find a distribution of search effort $\varphi=\{\varphi(i, t),(i, t) \in$ $\boldsymbol{K} \times \boldsymbol{T}$ \} maximizing the detection probability of the target. By the search plan $\varphi$, the probability that the target on path $\omega$ is detected is $1-\exp \left(-\sum_{t} \alpha_{\omega(t)} \varphi(\omega(t), t)\right)$ and therefore the detection probability $P(\varphi)$ which is the objective function is given by the following expression.

$$
P(\varphi)=1-\sum_{\omega \in \Omega} \pi(\omega) \exp \left(-\sum_{t=1}^{T} \alpha_{\omega(t)} \varphi(\omega(t), t)\right) .
$$

The function is finite and strictly concave for variables $\{\varphi(i, t),(i, t) \in S\}$ where $S \equiv$ $\{(\omega(t), t), t=1, \cdots, T, \omega \in \Omega\} \subseteq \boldsymbol{K} \times \boldsymbol{T}$. This problem is obtained by applying $c_{i t}=1$ and the objective function $P(\varphi)$ to the original problem $P_{M}$. The representation (13) of the optimal solution has the following analytic form in this problem.

$$
\varphi(i, t)=\left[\frac{1}{\alpha_{i}} \log \frac{\alpha_{i} \sum_{\omega \in \Omega_{i t}} \pi(\omega) \exp \left(-\sum_{\tau=1, \tau \neq t}^{T} \alpha_{\omega(\tau)} \varphi(\omega(\tau), \tau)\right)}{\lambda+\nu_{t}}\right]_{0}^{m_{i t}}
$$

where $\Omega_{i t}$ is defined as a set of target paths running through cell $i$ at time $t$, that is, $\Omega_{i t} \equiv\{\omega \in \Omega \mid \omega(t)=i\}$.

\subsection{Characteristics of the optimal distribution of search effort}

Here we elucidate some characteristics of the optimal distribution of search effort. The problem contains too many parameters to execute sensitivity analysis for all cases. We mainly focus the analysis on the effect of $\Phi(t)$ or $M$ on the optimal solution.

Basic case: The search space consists of $\boldsymbol{K}=\{1,2,3,4\}$ and $\boldsymbol{T}=\{1, \cdots, 5\}$ and the target selects one of 5 paths with equal probability $\pi(\omega)=1 / 5$. A set of target paths $\Omega$ is illustrated by Table 1 and Figure 1. 
Table 1 Target paths.

\begin{tabular}{cccccc}
\hline$\omega \backslash t$ & 1 & 2 & 3 & 4 & 5 \\
\hline 1 & 1 & 1 & 1 & 1 & 1 \\
2 & 2 & 2 & 2 & 2 & 2 \\
3 & 3 & 3 & 3 & 3 & 3 \\
4 & 1 & 2 & 3 & 4 & 4 \\
5 & 4 & 2 & 1 & 2 & 3 \\
\hline
\end{tabular}

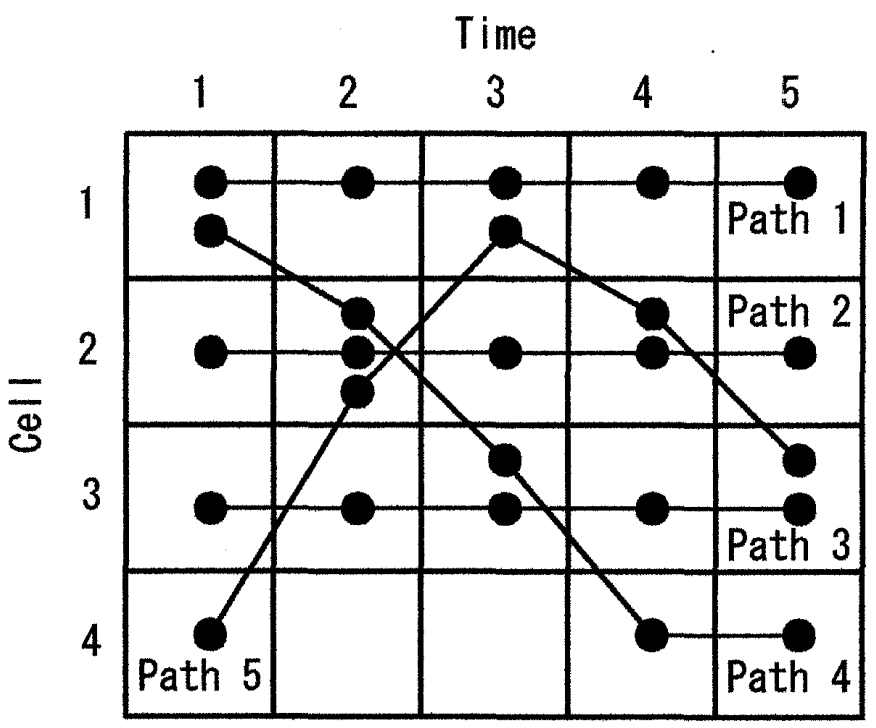

Figure 1 Target Paths.

Paths 1, 2 and 3 always stay on cells 1,2 and 3, respectively. On the other hand, Paths 4 and 5 move across the cell space right to left or left to right. The total search effort $M=5$ is available to the searcher on the whole search space and the subtotal effort $\Phi(t)=2$ at each time $t$. The amount of effort on every point is supposed to be unlimited, that is, $m_{i t}=\infty$. The detectability parameters are all $\alpha_{i}=0.2$ everywhere. Applying the gradient-completion and the total amount-completion methods to this problem, an optimal distribution of search effort is given as shown in Table 2. The maximum detection probability is $P\left(\varphi^{*}\right)=0.371$.

Table 2 Optimal distribution of search effort in basic case.

\begin{tabular}{ccccccc}
\hline Cell $\backslash t$ & 1 & 2 & 3 & 4 & 5 & Total \\
\hline 1 & 0.75 & 0 & 0.75 & 0 & 0 & \\
2 & 0 & 2.0 & 0 & 0 & 0 & \\
3 & 0 & 0 & 0.75 & 0 & 0.75 & \\
4 & 0 & 0 & 0 & 0 & 0 & \\
\hline Subtotal & 0.75 & 2.0 & 1.5 & 0 & 0.75 & 5.0 \\
\hline
\end{tabular}

In this basic case, Points $\{(i, t)\}=\{(1,1),(2,2),(1,3),(3,3),(2,4),(3,5)\}$ are crossing points of several paths, and should therefore be efficient points for search. Table 2 numerically supports the validity of this understanding. Three paths cross at $(2,2)$, which is why the local limit $\Phi(t)=2$ is concentrated on this point at time 2 . Paths 2 and 5 are probably detected by the $(2,2)$ search, so the detection efficiency of the point $(2,4)$ is relatively low and the searcher should not search at time 4 . This is the basic case.

By deleting the local limit $\Phi(t)$ from the basic case, we can estimate the unbiased efficiency of the detection for all points on the whole space. The obtained result is given in Table 3. The distributed effort of point $(2,2)$ increases to be $\varphi^{*}(2,2)=3.77$. In term of the unbiased detection efficiency, the point $(2,2)$ becomes very important. Focussing more effort on the point $(2,2)$ brings more detection probability $P\left(\varphi^{*}\right)=0.386$ than the basic case. 
Table 3 Optimal distribution of search effort(locally unlimited).

\begin{tabular}{ccccccc}
\hline Cell \t & 1 & 2 & 3 & 4 & 5 & Total \\
\hline 1 & 0.31 & 0 & 0.31 & 0 & 0 & \\
2 & 0 & 3.77 & 0 & 0 & 0 & \\
3 & 0 & 0 & 0.31 & 0 & 0.30 & \\
4 & 0 & 0 & 0 & 0 & 0 & \\
\hline Subtotal & 0.31 & 3.76 & 0.62 & 0 & 0.30 & 5.0 \\
\hline
\end{tabular}

The following is the case where more total effort $(M=12)$ is available than in the basic case. As shown in Table 4, some effort is distributed on point $(2,4)$, which is different from the above two cases. In this case, the marginal limit of search effort is $M_{\max }=10.0$. The optimal Lagrange multiplier $\lambda$ is 0 and the others are $\left\{\nu_{1}, \cdots, \nu_{5}\right\}=$ $\{0.037,0.042,0.034,0.029,0.031\}$. The maximum detection probability is $P\left(\varphi^{*}\right)=0.569$.

Table 4 Optimal distribution of search effort $(M=12)$.

\begin{tabular}{ccccccc}
\hline Cell \t & 1 & 2 & 3 & 4 & 5 & Total \\
\hline 1 & 2.0 & 0 & 0.65 & 0 & 0 & \\
2 & 0 & 2.0 & 0 & 2.0 & 0 & \\
3 & 0 & 0 & 1.35 & 0 & 2.0 & \\
4 & 0 & 0 & 0 & 0 & 0 & \\
\hline Subtotal & 2.0 & 2.0 & 2.0 & 2.0 & 2.0 & 10.0 \\
\hline
\end{tabular}

In the above three cases, crossing points of target paths play a critical role for search. However there might be a case that even the points which are estimated to be less important from the routing situation come to receive some effort. In the following case, the result of which is given in Table 5 , system parameters are set to be $M=10, \Phi(1)=\Phi(2)=\Phi(3)=$ $0.1, \Phi(4)=\Phi(5)=8.0$.

Table 5 Optimal distribution of search effort(early limit, $M=10$ ).

\begin{tabular}{ccccccc}
\hline Cell \t & 1 & 2 & 3 & 4 & 5 & Total \\
\hline 1 & 0.1 & 0 & 0 & 0.73 & 0.73 & \\
2 & 0 & 0.1 & 0 & 3.49 & 0 & \\
3 & 0 & 0 & 0.1 & 0 & 3.49 & \\
4 & 0 & 0 & 0 & 0.63 & 0.63 & \\
\hline Subtotal & 0.1 & 0.1 & 0.1 & 4.85 & 4.85 & 10.0 \\
\hline
\end{tabular}

Since the distribution of effort at time points $t=1,2,3$ is depressed, some effort is distributed on points $(1,4),(4,4),(1,5),(4,5)$ where only one path runs through. The detection probability is reduced to $P\left(\varphi^{*}\right)=0.464$ compared to the previous case, even though the same total amount of effort is expended.

\subsection{Computational time}

The original problem $P_{M}$ is a convex programming problem and can be solved by not only the proposed methods but also other well-known methods of non-linear programming. Here we compare the computational time of solving the problem by the proposed methods with that of the gradient projection method and the multiplier method.

As the experimental example, we take the two-dimensional search problem of the previous section. Problems are randomly generated as follows. First we decide the number of cells 
$K$, the number of time points $T$ and the number of target paths $|\Omega|$. A target path is constructed in such a way that a cell is randomly selected from $K$ cells at each of $T$ time points. The one-path-construction is repeated for each path, and we suppose $\pi(\omega)=1 /|\Omega|$. For the detectability parameter $\alpha_{i}$ of cell $i$, a real number is randomly chosen in the interval $[\underline{\alpha}, \bar{\alpha}]$. Finally, we set the limits of search effort $m_{i t}, \Phi(t), M$ and then the generation of a problem terminates. Through all computer experiments here, we set $|\Omega|=10, \underline{\alpha}=$ $0.1, \bar{\alpha}=0.5, M=5, \Phi(t)=1$ and $m_{i t}=6$ which means that the local limit $m_{i t}$ gives no practical constraint on the amount of search effort. We vary $K$ and $T$ of the space by $K=5,20(5), T=5,20(5)$ to measure CPU-times of solving many sizes of problems. Using a HITACHI S3600/120A mainframe and programming language FORTRAN 77, we solve each problem by four methods: the gradient-completion method, the total amountcompletion method, the gradient projection method and the multiplier method which are abbreviated to the GC method, the TAC method, the GP method and the M method for short, respectively. For each size, 50 problems are generated and solved by each of 4 methods. CPU-times are averaged for 50 problems and shown in Table 6. A symbol *** indicates the case that the algorithm did not terminate in 300 seconds. Approximately, problems larger $K=10$ and $T=10$ could not be solved by the M method, or larger than $K=20$ and $T=20$ by the GP method.

Table 6 CPU-times(sec) of four methods.

\begin{tabular}{cccccc}
\hline$K \backslash T$ & & 5 & 10 & 15 & 20 \\
\hline \multirow{4}{*}{5} & $G C$ & $5.3 \times 10^{-2}$ & $2.7 \times 10^{-1}$ & $4.7 \times 10^{-1}$ & $7.1 \times 10^{-1}$ \\
& $T A C$ & $4.0 \times 10^{-2}$ & $4.1 \times 10^{-1}$ & $8.3 \times 10^{-1}$ & $1.3 \times 10^{0}$ \\
& $G P$ & $1.9 \times 10^{-1}$ & $9.2 \times 10^{-1}$ & $2.6 \times 10^{0}$ & $5.9 \times 10^{0}$ \\
& $M$ & $7.9 \times 10^{0}$ & $2.6 \times 10^{+2}$ & $* * *$ & $* * *$ \\
\hline \multirow{4}{*}{10} & $G C$ & $8.9 \times 10^{-2}$ & $4.4 \times 10^{-1}$ & $7.8 \times 10^{-1}$ & $1.2 \times 10^{0}$ \\
& $T A C$ & $5.8 \times 10^{-2}$ & $7.6 \times 10^{-1}$ & $1.4 \times 10^{0}$ & $2.3 \times 10^{0}$ \\
& $G P$ & $1.1 \times 10^{0}$ & $6.2 \times 10^{0}$ & $2.3 \times 10^{+1}$ & $5.5 \times 10^{+1}$ \\
& $M$ & $6.0 \times 10^{+1}$ & $* * *$ & $* * *$ & $* * *$ \\
\hline \multirow{4}{*}{15} & $G C$ & $1.4 \times 10^{-1}$ & $6.5 \times 10^{-1}$ & $1.1 \times 10^{0}$ & $1.8 \times 10^{0}$ \\
& $T A C$ & $7.6 \times 10^{-2}$ & $1.1 \times 10^{0}$ & $1.9 \times 10^{0}$ & $3.7 \times 10^{0}$ \\
& $G P$ & $4.0 \times 10^{0}$ & $2.6 \times 10^{+1}$ & $7.7 \times 10^{+1}$ & $* * *$ \\
& $M$ & $* * *$ & $* * *$ & $* * *$ & $* * *$ \\
\hline \multirow{3}{*}{20} & $G C$ & $1.8 \times 10^{-1}$ & $6.6 \times 10^{-1}$ & $1.3 \times 10^{0}$ & $2.1 \times 10^{0}$ \\
& $T A C$ & $1.1 \times 10^{-1}$ & $1.3 \times 10^{0}$ & $2.5 \times 10^{0}$ & $4.3 \times 10^{0}$ \\
& $G P$ & $1.0 \times 10^{+1}$ & $6.1 \times 10^{+1}$ & $* * *$ & $* * *$ \\
& $M$ & $* * *$ & $* * *$ & $* * *$ & $* * *$ \\
\hline
\end{tabular}

From Table 6, we can summarize the tendency of the computational time for each method.

(1) The computational time of the M method is approximately $100 \sim 1000$ times as much as the proposed methods. The M method can be applied only to small problems.

(2) The GP method always expends more CPU-time than the proposed methods, especially for large $K$ and $T$. For small problems, its computational time is $3 \sim 10$ times as large as the proposed methods and $10 \sim 100$ times as large for large problems.

(3) Among the proposed two methods, superiority varies by the size of problem. On the whole, the GC method is superior to the TAC method. The increase of CPU-time with $K$ is low for both methods as seen from the result that the CPU-time for $K=20$ 
is only $2 \sim 3$ times than $K=5$. On the other hand, CPU-time increases rapidly with $T$. The CPU-time for $T=20$ is about 13 times that of $T=5$ for the GC method and $30 \sim 50$ times for the TAC method. This is the main reason why the GC method is superior to the TAC method for problems where $T$ is large.

The increase of $T$ directly causes the increase of the number of constraints $\Phi(t)$. For the execution of the algorithm, the number of subprocedure repetitions becomes larger and the convergence speed to an optimal solution becomes lower. Especially the TAC method has the algorithmic construction that subprocedure $A L_{\lambda}\left(\nu_{t}\right)$ is called from $A L_{\lambda}$ to temporarily obtain a convergence solution for each time. That might be the reason why the effect of increasing $T$ is steeper on the TAC method than the GC method.

\section{Conclusions}

This paper deals with a kind of two-dimensional resource allocation problem with double layers of constraints on the total amount of resources. From the viewpoint of the resource allocation problem, the problem is a generalized version which has a general concave objective function and doubly layered constraints. From the viewpoint of a non-linear problem, it is a specialized version which has a special form of constraints. We proposed two methods for the optimal solution. The proposed methods are constructed by making use of the simple relation between the constraints and their Lagrange multipliers. By numerical experiment, it is verified that the proposed methods perform better than other well-known methods. The necessary and sufficient conditions and the relation between the constraints and the Lagrange multipliers which we clarified in this paper could be extended on three or more dimensional space.

\section{Acknowledgment}

The authors express their appreciation to Professor A. R. Washburn at Naval Postgraduate School for helping them purify the first rude draft of this paper. The authors also thank some helpful comments of two anonymous referees and the associate editor.

\section{References}

[1] S.S. Brown: Optimal search for a moving target in discrete time and space. Operations Research, 28(1980) 1275-1289.

[2] J. de Guenin: Optimum distribution of effort: An extension of the Koopman basic theory. Operations Research, 9(1961) 1-9.

[3] R. Horst, P.M. Pardalos and N.V. Thoai: Introduction to Global Optimization (Kluwer Academic Publishers, London, 1995).

[4] R. Horst and H. Tuy: Global Optimization: Deteministic Approaches (Spring-Verlag, Berlin, 1996).

[5] T. Ibaraki and N. Katoh: Resource Allocation Problems: Algorithmic Approaches (The MIT Press, London, 1988).

[6] H. Konno and H. Yamasita: Nonlinear Programming (in Japanese) (Nikkagiren, Tokyo, 1984).

[7] B.O. Koopman: The theory of search III: The optimum distribution of searching effort. Operations Research, 5(1957) 613-626.

[8] O.L. Mangasarian: Nonlinear Programming (McGraw-Hill, New York, 1969).

[9] B. Martos: Nonlinear Programming: Theory and Methods (North Holland, Amsterdam, 1975). 
[10] R.T. Rockafellar: Convex Analysis (Princeton University Press, Princeton, 1970).

[11] A.R. Washburn: Search for a moving target: The FAB algorithm. Operations Research, 31(1983) 739-751.

\author{
Ryusuke Hohzaki \\ Department of Applied Physics \\ National Defense Academy \\ 1-10-20 Hashirimizu, Yokosuka, \\ 239-8686, Japan \\ E-mail: hozaki@cc.nda.ac.jp
}

